\title{
The breast lesion excision system procedure: An optimal solution for the management of indeterminate BI-RADS category 3 breast lesions in women with severe anxiety
}

Semih Hot, MD, PhD, Zafer Ü. Coşkun, MD, Prof, Adem Akçakaya, MD, Prof, Ömer Bender, MD, Prof, Ülkü A. Türkmen, MD, Prof, Pınar Ö. Nayır, MD, PhD, Ayhan Sarı, MD, PhD, Ayşe B. Hot, MD.

\begin{abstract}

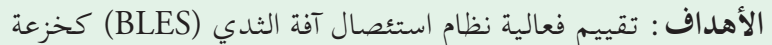

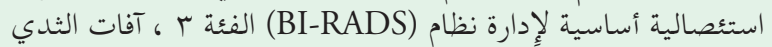
الصغيرة ، والصلبة عند الِّاء النساء اللواتي يعانين من القلق من سرطان آنات الثري الثدي.

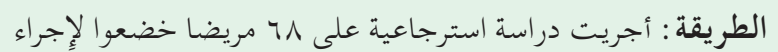
BLES

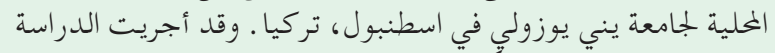

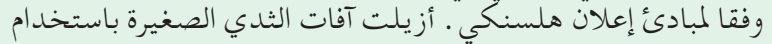

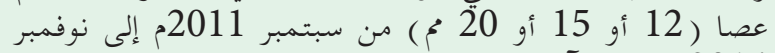

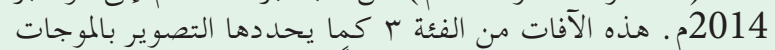

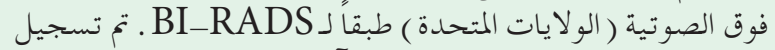

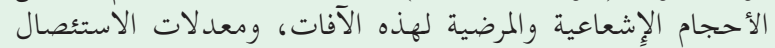

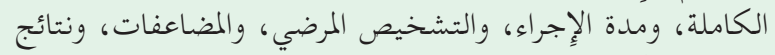
التصوير قبل وبعد العملية .

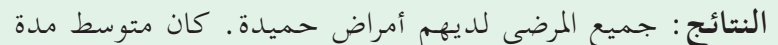

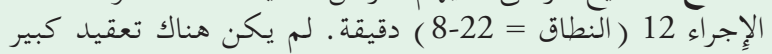

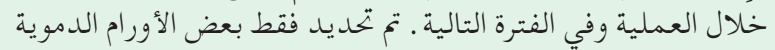

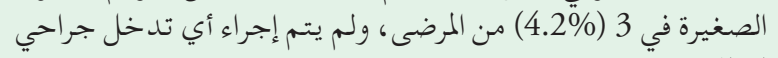
إضافي.

الخاتمة : إن إجراء BLES هو الحل الأمثل لإِدارة آفات الثدي الفئة 3

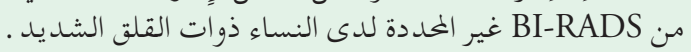

Objectives: To evaluate the efficacy of the Breast lesion excision system (BLES) procedure as a primary excisional biopsy for the management of breast imaging-reporting and data system (BI-RADS) category 3, small, and solid breast lesions in women having severe breast cancer anxiety.

Methods: A retospective study was conducted on 68 patients who underwent a BLES procedure. The study protocol was approved by the local ethical committee of Yeni Yuzyll University in Istanbul, Turkey. The study was carried out according to the principles of the Helsinki Declaration. Small breast lesions removed using a (12, 15 or $20 \mathrm{~mm}$ ) wand from September 2011 to November 2014. These were category 3 lesions as determined by ultrasound (US) imaging according to BI-RADS. The radiological and pathological sizes of these lesions, the complete excision rates, the procedure durations, the pathological diagnosis, the complications, and the imaging findings before and after the procedure were all recorded.

Results: All the patients had a benign pathology. The mean duration of procedure was 12 (range $=8-22$ ) minutes. There was no major complication during the procedure and in the following period. Only some small hematomas were determined in $3(4.2 \%)$ patients, and no additional surgical intervention was performed.

Conclusion: The BLES procedure is an optimal solution for the management of indeterminate BI-RADS category 3 breast lesions in women with severe anxiety.

Saudi Med J 2018; Vol. 39 (9): 891-896 doi: 10.15537/smj.2018.9.22573

From the Department of Surgery (Hot S), and from the Department of Pathology (Nayrr), Okmeydanı Education Research hospital, University of Health Science, from the Department of Surgery (Akçakaya), Faculty of Medicine, Bezmialem University, from the Vocational School of Health Services (Bender), Yeni Yüzynl University, from the Department of Psychology (Hot B), Bahçessehir University, Istanbul, from the Department of Radiology (Coskun), and from the Department of Anesthesiology and Reanimation (Türkmen), Faculty of Medicine, Giresun University, Giresun, and from the Department of Surgery (Sar)), Silvan Dr Yusuf Azizoğlu State Hospital, Ministry of Health, Diyarbakir, Turkey.

Received 1st April 2018. Accepted 1st August 2018.

Address correspondence and reprint request to: Dr. Semih Hot, Department of Surgery, Okmeydant Education Research Hospital, University of Health Science, Istanbul, Turkey. E-mail:semihhot@hotmail.com

ORCID ID: orcid.org/0000-0001-9623-356X 
$\mathrm{T}$ he assessment of BI-RADS category one and 2 (normal, and benign) and BI-RADS category 4 and 5 (suspected, and highly suspected) breast lesions are relatively easy for radiologists and clinicians; BI-RADS category 3 is more difficult. ${ }^{1}$ Many studies have shown that there are significant differences between radiologists who interpret breast lesions as BI-RADS $3 .^{2,3}$ In addition, radiologists who are not sure how to report a finding often extensively use BI-RADS 3 . However, BI-RADS 3 is moderate and has different meanings in US, mammography, and magnetic resonance imaging (MRI). Indeed, it is assessed differently in these 3 modalities. Breast imaging-reporting and data system 3 makes a large range of actions and reactions. It causes patient anxiety, removes some unnecessary biopsies, and is often overlooked by patients and clinicians. ${ }^{1}$

After a complete diagnostic assessment, classifying the imaging results as BI-RADS 3 is highly predictive of benignity, and experts allow for a short interval of follow-up rather than biopsy. ${ }^{1}$ However, the follow-up of BI-RADS category 3 indeterminate radiological lesions at 6-month intervals can create an issue for radiologists, patients, and breast surgeons. ${ }^{4}$ However, if the morphological characteristics of these lesions change over time (with delayed biopsy), then concerns arise that these lesions may progress to more advanced stages. ${ }^{4}$ Some mass lesions are biopsied due to concerns of breast cancer. ${ }^{5,6}$ Experts currently recommend imageguided biopsy of these lesions including fine-needle aspiration, percutaneous core biopsy, and vacuumassisted biopsy. ${ }^{7}$

The detection of a fibroadenoma (benign breast growth that most commonly presents in young women) can cause anxiety and concerns patients especially because the patients know that any breast lump could be cancerous. Fibroadenoma has a very slight risk of developing into breast cancer in the future, but the majority of fibroadenomas are benign breast lumps. ${ }^{8}$ However, sampling BI-RADS 3 small breast lesions under imaging guidance may result in an inadequate or non-definitive histological assessment. Therefore, the psychological distress and patient fear caused by the abnormal results of a mammography and the subsequent biopsy process may not be eliminated by negative biopsy results. ${ }^{5}$

Open surgical intervention for BI-RADS 3 intermediate breast lesions with local excision following

Disclosure. Authors have no conflict of interests, and the work was not supported or funded by any drug company. wire or radionuclide material findings is common. Alternative interventional radiological methods have recently been described and are now common for the excision of these lesions. These include vacuumassisted core needle biopsy (handheld Mammotome probe; Ethicon Endo-Surgery, Cincinnati, OH, USA) and intact BLES (Intact Medical Corp., Framingham, MA, USA) procedures. ${ }^{9,10}$ Generally, local anesthesia is sufficient to perform these procedures. The complication rate is quite low, and scar formation after the procedure occurs at a tolerable rate. Intact removal of these masses as a whole should always be preferred because if these masses lose their integrity, then the margin cannot be evaluated, and there may be some residual tissue left behind. ${ }^{11-13}$ Breast imaging-reporting and data system has been shown to be a safe and effective biopsy method used in thousands of cases in the United States of America for 15 years. ${ }^{10,14}$

Breast imaging-reporting and data system is an automated, vacuum-assisted single-pass biopsy device that uses radiofrequency (RF) with stereotactic or US guidance. It allows for a monoblock excision of breast lesions. This technique is an outpatient clinical procedure, and such RF cautery offers small but complete unfragmented excision. This intact architecture is sufficient for diagnosis and margin evaluation. The aim of this study was to evaluate the efficacy of BLES in excisional biopsy in women who had indeterminate BI-RADS 3 breast lesions of less than $2 \mathrm{~cm}$ in diameter and serious anxiety due to the possibility of breast cancer.

Methods. This retrospective study reviewed the records of women who underwent the BLES procedure between September 2011 and November 2014 in the interventional radiology unit of Okmeydanı Education Research hospital in Istanbul, Turkey, were reviewed. The local ethical committee of Yeni Yuzyll University in Istanbul, Turkey, approved the study protocol. The study was performed according to the principles of Helsinki Declaration. All patients underwent a BLES procedure using a $12-\mathrm{mm}, 15-\mathrm{mm}$, or $20-\mathrm{mm}$ probe. Patients older than 40 years of age were also evaluated with mammography to exclude obvious breast cancer cases. The procedure used US guidance on all patients by the same team. This team was composed of an experienced radiologist, breast surgeon, and anesthesia expert.

Excisional biopsy was performed with BLES in women who had moderate-severe anxiety due to the possibility of breast cancer and a BIRADS 3 breast lesion of $<2 \mathrm{~cm}$ in diameter as diagnosed by 
US. This procedure was not used in individuals with contraindications, chronic obstructive pulmonary disease, and cardiac failure. We excluded patients with less than $6 \mathrm{~mm}$ between the lesions or between the skin or chest wall under compression as well as those with a total compression thickness of the breast of less than $30 \mathrm{~mm}$. Positioning was performed very sensitively as soon as BLES was activated. Therefore, data on the first 10 patients who underwent the procedure were excluded from the study because the team had limited proficiency with the technique at that time. All patients were informed about the procedure and gave written, informed consents.

Breast lesion excision system may be performed under the guidance of MRI and US to increase the understanding of the treatment site (Figure 1). Touching a button turns on BLES; the metallic spikes stick out under US guided and surround the lesion. The probe and complete sample are then removed, and the specimen is sent to histology in formalin after radiographic evaluation. The external surfaces of the specimen are stained with ink, and cross-sections are taken perpendicular to the specimen's longest axis. These cross-sections are then serially embedded, and standard pathological analyses are performed until advanced pathological examinations can be conducted such as immunohistochemistry.

The known contraindications for the BLES procedure include patients with cardiac pacemakers or other RF devices because RF waves may potentially inhibit or damage the device. ${ }^{15}$ It is not recommended for pregnant women, and caution is required for bleeding

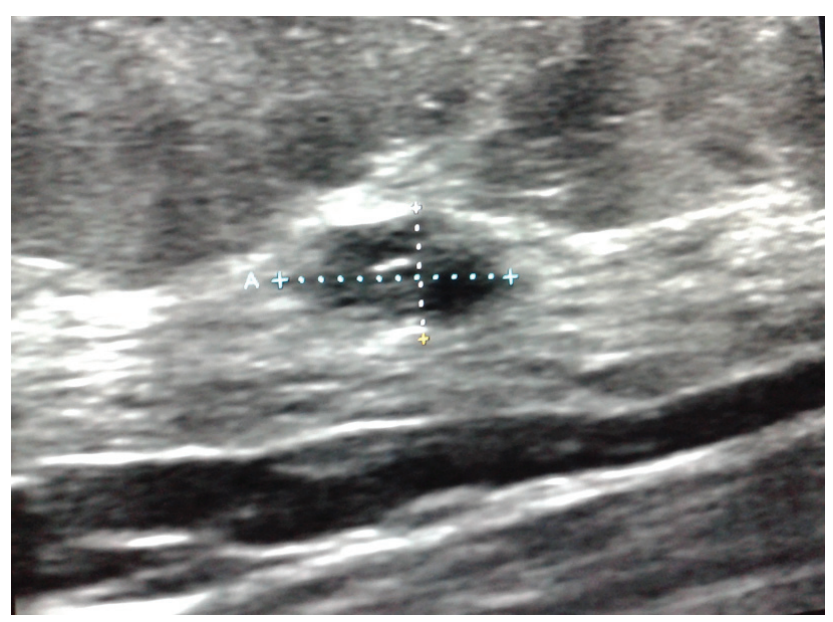

Figure 1 - Ultrasonographic image of the BLES (Intact Medical Corp., Framingham, MA, USA) procedure. control in individuals taking anticoagulants or those with coagulation defects. Before BLES application, careful attention should be given to the relationship between the skin surface and the chest wall of the lesion localization because RF waves transmitted from the metallic spikes may cause thermal burns and possible skin necrosis during biopsy. ${ }^{15}$ After lesion excision, a carbon-based absorbable tissue marker (BiomarC; Carbon Medical Technologies, Inc., St. Paul, MN, USA) was inserted using the same tract to identify the site in case further intervention was necessary. The mean absorption time of the marker was 6-months.

The Visual analogue scale (VAS) was administered in the procedure room to quantify the patients' pain. A VAS score of 0 indicates no pain, and 10 indicates the worst pain imaginable. Patients were followed for 3-5 hours after the procedure and then discharged. The same team performed the procedure on all patients and recorded the following: the radiological and pathological size of the lesions, the complete excision rate, the procedure duration, the pathological diagnosis, imaging findings before and after the procedure, and complications (allergic reaction, fainting, hematoma or seroma formation, thermal damage, wound infection, pain requiring analgesic use, delayed wound healing). An experienced breast pathologist performed the pathological examination.

The anxiety level of the patients was measured before the procedure and 6-months afterwards using the Hamilton anxiety rating scale (HAM-A) a widely used semi-structured scale developed for use in clinical and research settings to determine the severity of anxiety. Its validity and reliability have already been studied in Turkey. ${ }^{16}$ Each of the 14 items on the scale is defined by a series of symptoms. Psychological and physical anxiety is measured using the HAM-A test. ${ }^{17}$

Every item is evaluated on a measure between 0 (not available) and 4 (severe). The sum of the scores range from 0-56. Mild anxiety is less than 17 , and mildmoderate anxiety is between 18-24; moderate-severe anxiety is $25-30$. The BLES procedure was performed on patients who had a score of 25 points or more on the HAM-A questionnaire. Clinical and sonographic follow-ups were performed for all patients at 6-month intervals in the first year and once a year afterwards. An annual mammogram was added to the follow-up plan of patients older than 40 years of age. The mean follow-up period was 24-months (range=12-48 months).

This cross-sectional study investigated the efficacy of the BLES procedure in excisional biopsy in women with indeterminate BI-RADS 3 breast lesions of less than $2 \mathrm{~cm}$ in diameter. The statistical analyses used 
SPSS for Windows, Version 15.0 (SPSS Inc., Chicago, IL, USA). The VAS pain scores of the patients and the pathological distribution of lesions were analyzed using numbers, percentages, and standard deviation.

Results. A total of 70 BI-RADS category 3 indeterminate small breast lesions were found in the 68 patients over 3 years. The mean age of the patients who had excision biopsy was 48.3 years (range $=28-77$ years). All patients had a benign pathology. The complete excision ratio of the cases was $85 \%$ (60 of 70 lesions). Clear margin is not reported because all cases are benign.The mean duration of the procedure was 12 minutes (range $=8-22$ minutes). The VAS pain score questionnaire results of all patients showed that only 5 (7.1\%) patients complained of moderate discomfort; the remainder reported only mild discomfort (Figure 2). The median pain score was 3 out of 10 . There was no incidence of vasovagal syncope and no intolerant patients during procedure. Small hematomas were found in 3 (4.2\%) patients, but no additional surgical intervention was required to address this complication. There were no infected wounds. There was thermal damage in one of the first 10 cases; these were not included. In terms of device, 42 procedures were performed with a $20-\mathrm{mm}$ wand, 21 procedures with a $15-\mathrm{mm}$ wand, and 7 procedures with a $12-\mathrm{mm}$ wand.

The mean radiological width of the lesions was $16-\mathrm{mm}$ (range $=11-20 \mathrm{~mm}$ ). The pathological examination revealed minimal diathermia artifacts of

\section{VASPain Scores}

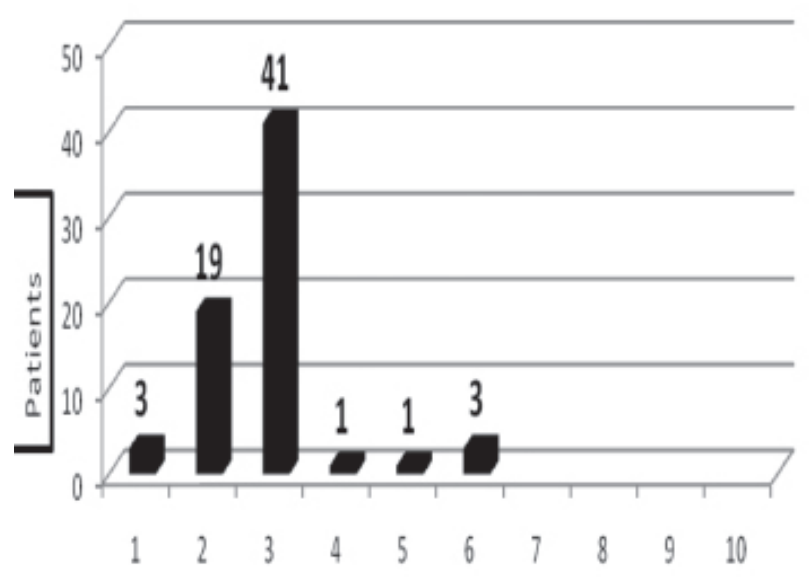

Figure 2 - Visual analogue scalepain scores of the patients. VAS - Visual analogue scale less than 1-mm on the margins of sample cross-sections, but these caused no problem in histology. The size of the lesions was correctly measured in cross-section, and the excision was sufficient in all cases apart from the definite diagnosis. The pathology results of all the lesions are summarized in (Table 1). The HAMA-A anxiety scores of all the patients at the control visit performed after 6-months were below 25 (Figure 3). These outcomes were accepted as very favorable results of the BLES procedure.

The patients were followed for a mean length of time of 24-months (range=12-48 months). No recurrence was observed during the follow-up period. Two years later, a breast mass was detected in the tissue of the other breast of one patient. This was also a BI-RADS category 3 lesion less than $2 \mathrm{~cm}$ in size; this lesion was also excised with a BLES procedure.

Discussion. Many articles have reported that fewer than $2 \%$ of BI-RADS 3 indeterminate lesions

Table 1 - Summary of final pathology.

\begin{tabular}{lcc}
\hline Lesion pathology & $\mathbf{n}$ & $(\%)$ \\
\hline Fibroadenoma & 40 & $(57.1)$ \\
Sclerosing adenosis & 14 & $(20)$ \\
Fibrocystic change & 7 & $(10)$ \\
Papilloma & 5 & $(7.1)$ \\
Granulomatous mastitis & 2 & $(2.8)$ \\
Tubular adenoma & 1 & $(1.4)$ \\
Lipogranuloma & 1 & $(1.4)$ \\
Total & 70 & $(100)$ \\
\hline
\end{tabular}

HAM-A SCORE VS. PATIENT

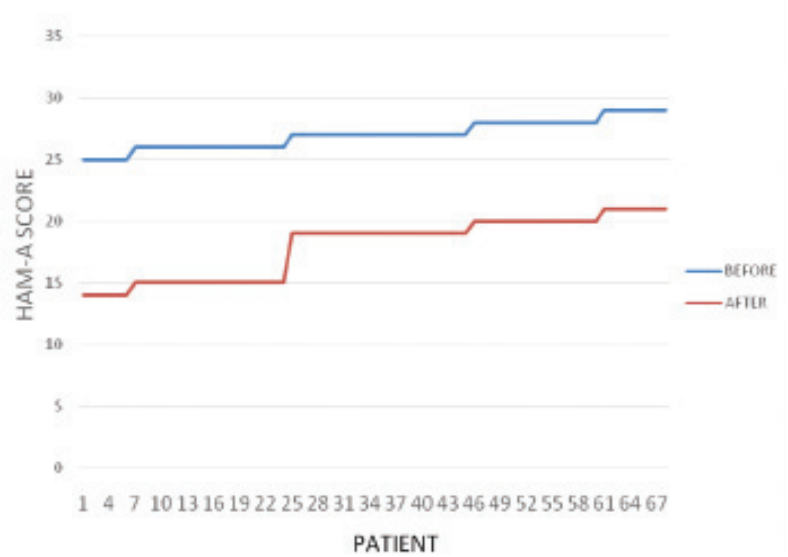

Figure 3 - Hamilton anxiety rating scale of the patients (before and after 6 months the procedure). HAMA-A - Hamilton anxiety rating scale 
were malignant after evaluation with additional mammography, diffusion weighted MRI, and US imaging. ${ }^{1,18}$ In our study, none of the patients had a malignant or premalignant lesion. Excision was preferred after wiring the lesion in one patient because there were microcalcification clusters on the mammography image. Patient compliance rates were low if additional imaging or only follow-up was recommended in patients with BI-RADS 3 indeterminate lesions. ${ }^{4}$ This compliance may decrease to $83.3 \%$ at 6 -months, $75.9 \%$ at 12 -months, and $53.9 \%$ within 2 years. ${ }^{4}$ These decreases are significant because follow-up at short intervals covers not only the first 6-months but also up to 2 years. There was only one patient who had BLES excision who did not attend the follow-up visits. This patient was excluded from the study.

Breast cancer is the most commonly encountered cancer among women in developed and developing countries. Globally, more than 570,000 women died from breast cancer in 2015. ${ }^{19}$ Mammography screening studies can decrease mortality due to breast cancer. ${ }^{20}$ However, screening and diagnostic tests frequently cause anxiety in women when breast lesions are observed, this decreases the quality of life. ${ }^{21}$ Therefore, the minimally invasive BLES technique was used for these women with severe anxiety due to breast masses. The patients did not have to live with a mass in their breast or observe long-term follow-up protocols. We found that the anxiety level was significantly decreased when the pathology results indicated benign lesions completely removed via a tolerable procedure under local anesthesia.

Grady et $\mathrm{al},{ }^{11}$ demonstrated that there was no need for an additional surgical intervention or follow-up procedure after complete excision of $<2 \mathrm{~cm}$ diameter lesions. We found 70 indeterminate breast lesions in 68 patients who were anxious due to diagnosis with BI-RADS category 3 masses $(<2 \mathrm{~cm}$ diameter). These subjects underwent total excision with the BLES procedure. Breast lesion excision system is an effective and well-tolerated biopsy procedure with few complications. It facilitates correct pathological examination and offers a whole specimen. However, in addition to these contraindicated conditions, this procedure is not suitable for lesions that are close to the skin, in cases with multiple lesions, or for women with small breast tissue.

Seror et $\mathrm{al}^{, 22}$ and Allen et $\mathrm{al},{ }^{15}$ reported only one hematoma in prospective cohorts of 163 and 74 patients. Sie et al, ${ }^{10}$ and Killebrew et al, ${ }^{14}$ had larger retrospective cohorts. Sie at $\mathrm{al},{ }^{10}$ described one infection that was treated with oral antibiotics. Minor complications may have occurred without being recorded. Lower complication rates were reported in previous studies. The exclusion of procedures performed during the introductory period might explain this lower rate. Sie et al, ${ }^{10}$ excluded the first 15 patients at each research site.

We found no early or late complications other than 3 small hematomas; no additional surgical intervention was performed in the hematoma cases. Moreover, all specimens obtained using the BLES technique had excellent quality for pathological analysis. The remaining tissue with low-grade malignancy left after vacuum excision of a papilloma or any remaining borderline lesions can lead to serious negative outcomes. There is no defined margin for excision, and the value of extended vacuum biopsy is definitely debatable for indeterminate and potentially malign lesions. Breast lesion excision system clearly prevents this problem, and it provides a small but safe margin. The papilloma literature has only a few small case series and a limited number of articles with only short-term findings. ${ }^{15,23}$ Although the size of the available wands precludes the excision of many lesions, benign lesions with a suitable size and localization can be effectively treated in an outpatient clinic. The BLES procedure can excise the small $(\leq 10$ $\mathrm{mm}$ ) breast carcinomas without fragmentation and can provide a clear margin. ${ }^{24}$ However these carcinomas must be solid lesions that can be seen radiologically. The BLES procedure has a therapeutic role in these cases. A clear biopsy margin should be $1-\mathrm{mm}$ and over. This is the only independent predictor of complete lesion excision. ${ }^{24}$

This study has some limitations. The individual breast cancer risk of the patients was not calculated, and breast MRI was not performed. A breast MRI and an individual breast cancer risk calculation can change the patients' anxiety levels. Thus, the selection of the patients for the BLES procedure would improve. However, 6-months lesion follow-up would remain an issue.

In conclusion, these results indicate that BLES is a beneficial biopsy tool for women with BI-RADS 3 indeterminate breast lesions and severe anxiety. It can be performed in an outpatient clinic setting with low complication rates, minimal scar tissue, and complete mass removal. There is a need for long-term studies comparing this technique with control subjects so that the procedure can be accepted as the gold standard in the management of indeterminate BI-RADS category 3 small breast lesions.

Acknowledgment. The authors would like to thank the American Manuscript Editing Company for its English Editing Service. 


\section{References}

1. Lee KA, Talati N, Oudsema R, Steinberger S, Margolies LR. BI-RADS 3: Current and Future Use of Probably Benign. Curr Radiol Rep 2018; 6: 5.

2. Michaels AY, Chung CSW, Frost EP, Birdwell RL, Giess CS. Interobserver variability in upgraded and non-upgraded BI-RADS 3 lesions. Clin Radiol 2017; 72: e1-e6.

3. Grimm LJ, Anderson AL, Baker JA, Johnson KS, Walsh $\mathrm{R}$, Yoon SC, et al. Interobserver Variability Between Breast Imagers Using the Fifth Edition of the BI-RADS MRI Lexicon. AJR Am J Roentgenol 2015; 204: 1120-1124.

4. Chung CS, Giess CS, Gombos EC, Frost EP, Yeh ED, Raza $S$, et al. Patient compliance and diagnostic yield of 18-month unilateral follow-up in surveillance of probably benign mammographic lesions. AJR Am J Roentgenol 2014; 202: 922-927.

5. Kamath J, Cruess DG, Claffey K, Wilson L, Phoenix N, Tannenbaum S. Symptom distress associated with biopsy in women with suspect breast lesions. ISRN Oncol 2012; 2012: 898327.

6. Ha R, Kim H, Mango V, Wynn R, Comstock C. Ultrasonographic features and clinical implications of benign palpable breast lesions in young women. Ultrasonography 2015; 34: 66-70.

7. Hoffmann O, Stamatis GA, Bittner AK, Arnold G, Schnabel $\mathrm{R}$, Krüger K, et al. B3-lesions of the breast and cancer risk - an analysis of mammography screening patients. Mol Clin Oncol 2016; 4: 705-708.

8. Wu YT, Chen ST, Chen CJ, Kuo YL, Tseng LM, Chen DR, et al. Breast cancer arising within fibroadenoma: collective analysis of case reports in the literature and hints on treatment policy. World J Surg Oncol 2014; 12: 335.

9. Medjhoul A, Canale S, Mathieu MC, Uzan C, Garbay JR, Dromain C, et al. Breast lesion excision sample (BLES biopsy) combining stereotactic biopsy and radiofrequency: is it a safe and accurate procedure in case of BIRADS 4 and 5 breast lesions? Breast J 2013; 19: 590-594.

10. Sie A, Bryan DC, Gaines V, Killebrew LK, Kim CH, Morrison $\mathrm{CC}$, et al. Multicenter evaluation of the breast lesion excision system, a percutaneous, vacuum-assisted, intact-specimen breast biopsy device. Cancer 2006; 107: 945-949.

11. Grady I, Gorsuch H, Wilburn-Bailey S. Long-term outcome of benign fibroadenomas treated by ultrasound-guided percutaneous excision. Breast J 2008; 14: 275-278.

12. Tagaya N, Nakagawa A, Ishikawa Y, Oyama T, Kubota K. Experience with ultrasonographically guided vacuum-assisted resection of benign breast tumors. Clin Radiol 2008; 63: 396-400.
13. Ko EY, Bae YA, Kim MJ, Lee KS, Lee Y, Kim LS. Factors affecting the efficacy of ultrasound-guided vacuum-assisted percutaneous excision for removal of benign breast lesions. $J$ Ultrasound Med 2008; 27: 65-73.

14. Killebrew LK, Oneson RH. Comparison of the diagnostic accuracy of a vacuum-assisted percutaneous intact specimen sampling device to a vacuum-assisted core needle sampling device for breast biopsy: initial experience. Breast J 2006; 12 : 302-308.

15. Allen SD, Nerurkar A, Della Rovere GU. The breast lesion excision system (BLES): a novel technique in the diagnostic and therapeutic management of small indeterminate breast lesions? Eur Radiol 2011; 21: 919-924.

16. Turgut GE, Yüksel BC, Polat E, Yıldız Y, Berkem H, Özel $\mathrm{H}$, et al. The affecting factors on anxiety in female patients with breast complaints. The Journal of Breast Health 2009; 5: 92-97.

17. Bandelow B, Michaelis S, Wedekind D. Treatment of anxiety disorders. Dialogues Clin Neurosci 2017; 19: 93-107.

18. Lee S, Jung Y, Bae Y. Synchronous BI-RADS Category 3 Lesions on Preoperative Ultrasonography in Patients with Breast Cancer: Is Short-Term Follow-Up Appropriate? J Breast Cancer 2015; 18: 181-186.

19. Breast Cancer. Cancer [Internet]. World Health Organization: Geneva (CH); 2018 .[cited 2018 Feb 15]. Available from: http://www.who.int/cancer/prevention/diagnosis-screening/ breast-cancer/en.

20. Mandelblatt J, van Ravesteyn N, Schechter C, Chang Y, Huang AT, Near AM, et al. Which strategies reduce breast cancer mortality most? Collaborative modeling of optimal screening, treatment, and obesity prevention. Cancer 2013; 119: 2541-2548.

21. Fuller MS, Lee CI, Elmore JG. Breast cancer screening: an evidence-based update. Med Clin North Am 2015; 99: 451-468.

22. Seror JY, Lesieur B, Scheuer-Niro B, Zerat L, Rouzier R, Uzan S. Predictive factors for complete excision and underestimation of one-pass en bloc excision of non-palpable breast lesions with the Intact $\left({ }^{\oplus}\right)$ breast lesion excision system. Eur J Radiol 2012; 81: 719-724.

23. Wei H, Jiayi F, Qinping Z, Junyi S, Yuan S, Li L, et al. Ultrasound-guided vacuum-assisted breast biopsy system for diagnosis and minimally invasive excision of intraductal papilloma without nipple discharge. World J Surg 2009; 33: 2579-2581.

24. Papapanagiotou IK, Koulocheri D, Kalles V, Liakou P, Michalopoulos NV, Al-Harethee W, et al. Margin-free excision of small solid breast carcinomas using the Intact Breast Lesion Excision System ${ }^{\oplus}$ : is it feasible? Breast Cancer 2018; 25: 134-140. 